\title{
Agricoltura, paesaggio e territorio tra conservazione e innovazione: il ruolo della ricerca
}

Il paesaggio è un'entità spaziale complessa, nella quale troviamo registrate, sedimentate nel tempo, le trasformazioni di genere naturale e culturale, espresse con un linguaggio universalmente comprensibile in un testo unico e fragile.

A questa concezione matura del paesaggio si può riferire il suo riconoscimento come indicatore di sintesi dello stato dell'ambiente, che l'ex Agenzia nazionale per la protezione dell'ambiente proponeva sul finire degli anni Novanta, indicando pertanto una possibile via, non semplice, né esaustiva, ma chiara e praticabile, per l'analisi e il progetto della sostenibilità dello sviluppo territoriale.

La stessa definizione olistica che la Convenzione europea attribuisce al paesaggio costituisce una conferma ed un rafforzamento del suo significato quale esito primario delle modalità di sviluppo territoriale e pertanto della sua valenza di termine di valutazione delle relative condizioni di sostenibilità.

Il Codice dei beni culturali e del paesaggio prescrive la definizione di linee di sviluppo territoriale coerenti con i distinti caratteri strutturali propri dei paesaggi della nazione e con il principio del minore consumo di suolo, estendendo esplicitamente alle aree agricole congrue attenzioni tradizionalmente rivolte alle aree di riconosciuta sensibilità.

Si tratta di principi che promuovono con forza un concreto passaggio dalle discussioni alle azioni, che, pur non prevedendo l'eliminazione delle prime, comporta una maggiore diffusione delle seconde, finora troppo rare per produrre risultati soddisfacenti.

Al complesso ruolo contemporaneo riconosciuto al paesaggio ed all'agricoltura corrispondono importanti declinazioni delle competenze disciplinari ed essenziali esigenze di interazioni transdisciplinari ormai affermate dallo stesso sviluppo degli studi delle scienze agrarie e del paesaggio.

Gli orientamenti strategici emanati dall'Unione Europea per i territori rurali sono espressamente riferiti agli obiettivi di sostenibilità dello sviluppo già fissati nel 2001 e prevedono fra le aree tematiche prioritarie dell'asse ambientale della nuova politica agricola la conservazione della biodiversità espressa dai paesaggi rurali e della loro specifica connotazione culturale storica, nonché la tutela delle risorse ambientali ed il miglioramento della qualità della vita.

L'AISSA, che rappresenta le Società scientifiche che si occupano di ricerca in agricoltura, ha ritenuto pertanto di interesse proporre in occasione del suo annuale convegno nazionale un momento di convergenza di ricerca di diverso approccio e di diversa estrazione tecnicoscientifica sulla questione di ampio interesse pubblico della sostenibilità dello sviluppo territoriale, considerando il paesaggio come possibile destinatario comune delle molteplici attività conoscitive e progettuali che sono maturate e di quelle che si vanno sviluppando.

Zeno Varanini Presidente AISSA 